\title{
Studies of Nucleon Form Factors with 12 GeV CEBAF and SuperBigBite
}

\author{
J.-O. Hansen, \\ for the Jefferson Lab E12-07-109, E12-09-016 and E12-09-019 collaborations ${ }^{1}$
}

Jefferson Lab, 12000 Jefferson Avenue, Newport News, VA 23606, USA

\begin{abstract}
The elastic electromagnetic form factors are among the most fundamental quantities that describe the ground-state structure of the proton and neutron. Precision data of the form factors over a wide kinematical range provide a powerful test of current theories of hadron structure. A number of experiments aiming to measure the electric and magnetic elastic form factors of the neutron, $G_{E}^{n}$ and $G_{M}^{n}$, and proton, $G_{E}^{p}$, at very high momentum transfer, up to the range of $Q^{2}=10-14(\mathrm{GeV} / \mathrm{c})^{2}$, are planned to be carried out with the future $11 \mathrm{GeV}$ electron beam of the upgraded CEBAF at Jefferson Lab. These experiments will determine the nucleon form factors with unprecedented precision to $Q^{2}$-values up to three times higher than those of existing data. We review the approved proposals and the conceptual design of a new spectrometer, SuperBigBite, that will be used in these and other future experiments at Jefferson Lab.
\end{abstract}

Keywords: Electromagnetic form factors; polarization in interactions and scattering; elastic and quasi-elastic electron scattering; polarized targets; magnetic spectrometers; tracking detectors

PACS: 13.40.Gp; 13.88.+e; 14.20.Dh; 25.30.Bf; 25.30.Dh; 29.25.Pj; 29.30.Aj; 29.40.Gx

\section{INTRODUCTION}

The nucleon electromagnetic form factors have long been recognized as important benchmark quantities for testing models of the nucleon [1]. As they describe the ground-state nucleon structure, they manifestly relate to physics in the complex non-perturbative QCD regime that has proven difficult to treat theoretically. Additionally, they provide important model-independent constraints on General Parton Distribution (GPD) sum rules and are attractive targets for lattice QCD calculations. They also play an important role in the analysis of a variety of other nuclear physics experiments, such as parity-violating electron scattering and nuclear medium effect studies.

The electric and magnetic form factors, $G_{E}$ and $G_{M}$, of the nucleon can be measured in elastic electron-nucleon scattering. Since no free neutron targets exist, one obtains the neutron form factors via quasi-elastic scattering from light nuclear targets such as deuterium or ${ }^{3} \mathrm{He}$. Interest in precise nucleon form factor measurements has greatly intensified over the past decade, not least due to the discovery by Jones et al. [2] that the ratio of the proton's electric to magnetic form factors, $G_{E}^{p} / G_{M}^{p}$, decreases almost linearly from unity with increasing four-momentum transfer $Q^{2}$. This effect is not well understood to date, despite a wealth of theoretical work attempting to explain it.

Existing data for $G_{E}^{p} / G_{M}^{p}$ are shown elsewhere in this volume [3] and in the literature [4]. The almost linear downward trend of the ratio with $Q^{2}$ can be clearly seen, although the highest data points with their increasingly lower precision would also be consistent with a slowing drop-off, as some theoretical models suggest. Other models predict a continued linear decrease. Irrespective of the functional dependence, theory generally seems to favor a sign change of $G_{E}^{p}$ between $Q^{2}$ of approximately 6 and $14(\mathrm{GeV} / \mathrm{c})^{2}$, a remarkable yet not completely unexpected result [5]. Clear experimental evidence of this behavior would obviously be of tremendous import to the field.

Motivated by the intriguing observations about the proton form factors and by the advent of improved experimental capabilities, the precise measurement of the neutron form factors has also become a focus of renewed experimental activity in recent years. A summary of existing data for the neutron form factor ratio $G_{E}^{n} / G_{M}^{n}$ is published elsewhere [6]. One notes a stark divergence of the various theoretical models for this observable already at relatively low $Q^{2}$, starting approximately in the region of the highest measured datum at $Q^{2}=3.5(\mathrm{GeV} / \mathrm{c})^{2}$. As with the proton, there is a suggestion that $G_{E}^{n}$ may become negative at very high $Q^{2}$ [7], although theory is less consistent here. Neutron form factor data in the same high- $Q^{2}$ region as those available for the proton will allow a crucial cross-check of the theories

\footnotetext{
${ }^{1}$ For the full collaboration author lists, please see the respective experiment links under [12].
} 
that have been advanced to explain the behavior of the proton form factors. Hence, a precision measurement of $G_{E}^{n}$ in the region $Q^{2} \approx 10(\mathrm{GeV} / \mathrm{c})^{2}$ would be extremely valuable

A primary goal of future form factor experiments, then, should be to carry out a program of measurements for both the proton and the neutron in the essentially unexplored region of high $Q^{2} \gtrsim 7(\mathrm{GeV} / \mathrm{c})^{2}$ while maintaining the highest precision possible. Such measurements will discriminate cleanly between most of the various competing models currently on the market and thus offer much-needed insight into the short-range physics of the nucleon ground state. In view of the unexpected results already found, further discoveries would not be surprising.

\section{PROPOSED MEASUREMENTS}

The $12 \mathrm{GeV}$ upgrade of the Continuous Electron Beam Accelerator Facility (CEBAF) at Jefferson Lab, expected to be completed by 2014, will provide excellent experimental conditions for precision form factor measurements at high $Q^{2}$. The facility will be able to deliver a polarized electron beam of up to $11 \mathrm{GeV}$ energy to the existing halls with $I \approx 100 \mu \mathrm{A}$ current and $85 \%$ polarization, permitting the design of very high luminosity experiments.

Precision data of the nucleon electric form factors, $G_{E}^{p}$ and $G_{E}^{n}$, is obtained primarily through polarization experiments. Unlike cross section measurements, where the form factors enter quadratically into observables, the sensitivity to the less dominant electric form factors is greatly enhanced in polarization experiments as the form factors appear linearly in spin-dependent interference terms. Asymmetry measurements also offer vastly better control over systematics. The current best measurement techniques are polarized target experiments, $\vec{A}\left(\vec{e}, e^{\prime} N\right) X$, and recoil polarization experiments, $A\left(\vec{e}, e^{\prime} \vec{N}\right) X$, where $A$ is an appropriate target (hydrogen, deuterium, or ${ }^{3} \mathrm{He}$ ) and $N$, the corresponding nucleon of interest, either $p$ or $n$. The figure of merit of the former reaction is proportional to $P_{t}^{2} E^{2} / Q^{12}$, where $E$ is the beam energy and $P_{t}$, the target polarization. For the latter technique, it is proportional to $A_{y}^{2} E^{2} / Q^{12} \propto E^{2} / Q^{16}$, where $A_{y} \propto 1 / p_{\text {recoil }} \propto 1 / Q^{2}$ is the polarimeter analyzing power. Hence, it is generally more favorable at high $Q^{2}$ to do polarized target experiments. On the other hand, the current generation of polarized proton targets cannot handle high beam currents; therefore, for proton measurements, the polarization transfer reaction with an unpolarized high-density target is the better choice if high beam current is available and the detectors can withstand high rates.

Based on these considerations and primarily driven by Wojtsekhowski [8], several nucleon form factor experiments have been proposed and approved for Jefferson Lab's Hall A:

- E12-07-109: Measurement of $G_{E}^{p} / G_{M}^{p}$ using the recoil polarization technique. Three kinematics are proposed between $Q^{2}$ of 5 and $12(\mathrm{GeV} / \mathrm{c})^{2}$, with overall absolute uncertainties of the ratio ranging from $0.025-0.065$.

- E12-09-016: Measurement of $G_{E}^{n} / G_{M}^{n}$ using the double-polarization technique with a polarized ${ }^{3} \mathrm{He}$ target. Again, three kinematics are proposed between $Q^{2}$ of 5 and $10(\mathrm{GeV} / \mathrm{c})^{2}$, with an expected overall absolute uncertainty of the form factor ratio of better than 0.06 for each data point.

- E12-09-019: Measurement of $G_{M}^{n} / G_{M}^{p}$ up to $Q^{2}=13.5(\mathrm{GeV} / \mathrm{c})^{2}$ using the ratio of the $d\left(e, e^{\prime} n\right)$ to $d\left(e, e^{\prime} p\right)$ unpolarized cross-sections, a technique successfully employed in various previous experiments [9]. The expected combined relative uncertainty on $G_{M}^{n}$ is between 1.25 and $2.65 \%$, exceeding the precision of an approved complementary Hall B experiment [10].

These three experiments will share a common set of instrumentation, collectively referred to as SuperBigBite (see below). A fourth form factor experiment is planned in Hall A, which is not part of the SuperBigBite project: E1207-108, an absolute measurement of $G_{M}^{p}$ with a precision of $1-2 \%$ up to $Q^{2}=15.5(\mathrm{GeV} / \mathrm{c})^{2}$ [11]. With an absolute $G_{M}^{p}$ calibration at hand, it will be possible to extract absolute form factors from the measured ratios. Details about the kinematics and other experimental parameters of these experiments can be found in the respective proposals [12].

\section{INSTRUMENTATION}

SuperBigBite will be set of components allowing for flexible configuration of different experiments. One common feature of the proposed form factor experiments is the need for a medium acceptance hadron spectrometer at forward angles suitable for a high rate environment. These goals can be accomplished with a large open-geometry apparatus 
and GEM tracker technology. SuperBigBite includes the following key components [13]:

- A large dipole magnet with $2.5 \mathrm{Tm}$ field integral. It provides vertical bending and a large acceptance of up to $70 \mathrm{msr}$ at $15^{\circ}$. A Lambertson-style cutout allows the magnet to be rotated to central scattering angles of as little as $3.5^{\circ}$. The expected momentum, angular, and vertex resolutions are $0.5-1 \%, 0.3 \mathrm{mrad}$, and $1-2 \mathrm{~mm}$, respectively.

- GEM tracking chambers with a rate capability of up to $100 \mathrm{MHz} / \mathrm{cm}^{2}$ and better than $70 \mu \mathrm{m}$ position resolution. These trackers are based on the successful COMPASS design [14], but will have a larger size of $40 \times 50 \mathrm{~cm}^{2}$ instead of $31 \times 31 \mathrm{~cm}^{2}$. They are expected to be operated at a maximum rate of $500 \mathrm{kHz} / \mathrm{cm}^{2}$.

- A large hadron calorimeter with $5.5 \mathrm{~m}^{2}$ area, $1.5 \mathrm{~ns}$ time and $2 \mathrm{~cm}$ position resolution.

- $\mathrm{CH}_{2}$ analyzers for the proton polarimeter.

While this instrumentation will serve primarily for hadron detection, it will be used in combination with existing equipment, such as the BigBite spectrometer [15] or the BigCal calorimeter for electron detection. All experiments measure the (quasi-)elastically scattered electron and recoil nucleon in coincidence to select the elastic reaction channel. Trigger rates can be kept low by requiring a high threshold in the hadron calorimeter.

The $G_{E}^{p}$ experiment will employ a standard $40 \mathrm{~cm}$ long liquid hydrogen target, providing a luminosity of $8 \cdot 10^{38}$ $\mathrm{cm}^{-2} \mathrm{~s}^{-1}$. Conversely, the $G_{E}^{n}$ experiment will rely on polarized ${ }^{3} \mathrm{He}$ target that acts as an effective polarized neutron target. Using a novel thermal convection technique, the ${ }^{3} \mathrm{He}$ target is expected to achieve a polarization of over $65 \%$ at $40 \mu \mathrm{A}$ beam current. With a $60 \mathrm{~cm}$ long target cell, a luminosity of $6 \cdot 10^{36} \mathrm{~cm}^{-2} \mathrm{~s}^{-1}$ can be reached. Finally, the $G_{M}^{n}$ ratio-method measurement will use a standard $10 \mathrm{~cm}$ liquid deuterium target at a luminosity of $3 \cdot 10^{37} \mathrm{~cm}^{-2} \mathrm{~s}^{-1}$.

\section{CONCLUSIONS}

In summary, the SuperBigBite project in Jefferson Lab's Hall A is expected to measure the nucleon elastic form factors at the highest $Q^{2}$ to date with unprecedented precision of typically a few percent. The measurements represent the current state of the art in that they have the best achievable figures of merit in each case. The results will provide very interesting input for theory in a previously largely unexplored kinematical region. They promise a significant advancement of our understanding of the nucleon ground state.

\section{ACKNOWLEDGMENTS}

The author is grateful to Brian Quinn for helpful comments on the manuscript. This work was supported by DOE contract DE-AC05-06OR23177, under which Jefferson Science Associates, LLC, operates Jefferson Lab.

\section{REFERENCES}

1. J. Arrington, K. de Jager and C. F. Perdrisat, J. Phys. Conf. Ser. 299, 012002 (2011).

2. M. K. Jones et al., Phys. Rev. Lett. 84, 1398 (2000).

3. C. F. Perdrisat, "Final Results of the GEp(III) Experiment at Jefferson Lab," in Proceedings of the 19 ${ }^{\text {th }}$ Particles and Nuclei International Conference (PANIC11), edited by G. Stephans and F. Taylor, AIP Conf. Proc. (this volume).

4. A. J. R. Puckett et al., Phys. Rev. Lett. 104, 242301 (2010); arXiv:1102.5737v2, March 2011, submitted to Phys. Rev. C.

5. N. Dombey, Rev. Mod. Phys. 41, 236 (1969).

6. S. Riordan et al., Phys. Rev. Lett. 105, 262302 (2010).

7. I. C. Cloët et al., Few Body Syst. 46, 1 (2009).

8. B. Wojtsekhowski, "Nucleon Form Factor Experiments with 12 GeV CEBAF," AIP Conf. Proc. 1056, 365 (2008).

9. J. Lachniet et al., Phys. Rev. Lett. 102, 192001 (2009).

10. W. Brooks, G. Gilfoyle, J. Lachniet and M. Vineyard, spokespersons, "Measurement of the Neutron Magnetic Form Factor at High $Q^{2}$ Using the Ratio Method on Deuterium,” Jefferson Lab PAC32 Proposal PR12-07-104, June 2007.

11. B. Moffit, S. Gilad, B. Wojtsekhowski and J. Arrington, spokespersons, "Precision Measurement of the Proton Elastic Cross Section at High $Q^{2}$," Jefferson Lab PAC32 Proposal PR12-07-108, June 2007.

12. List of approved Hall A $12 \mathrm{GeV}$ proposals, http://www . jlab.org/exp_prog/generated/12GeV/halla.html.

13. C. W. de Jager et al., "The SuperBigBite Spectrometer for Jefferson Lab Hall A," Conceptual Design Report, 2009 (unpubl.).

14. B. Ketzer et al., Nucl. Phys. B (Proc. Suppl.) 125, 368 (2003).

15. D. W. Higinbotham, "BigBite Spectrometer," in Hall A Status Report 2008, edited by by C. W. de Jager and B. Moffit, 2009, pp. 28-32, available at http://hallaweb.jlab.org/publications/AnnualReports/. 